\title{
Étude sérologique des souches marocaines d'Agrobacterium tumefaciens, agent de la galle du collet des rosacées fruitières
}

\author{
A Benjama ${ }^{1 *}$, N Alami ${ }^{2}$ EM Saadaoui 2 \\ 1 Laboratoire de phytobactériologie, Inra, Marrakech, BP 533; \\ 2 Département de biologie, faculté des sciences, Meknès, Maroc
}

(Reçu le 29 avril 1996 ; accepté le 23 septembre 1996)

\begin{abstract}
Summary - Serological study of Moroccan strains of Agrobacterium tumefaciens, the causal agent of crowngall in stone-fruit trees. To investigate the possibility of using serological diagnosis methods for Agrobacterium tumefaciens, the causal agent of crown-gall of stone-fruit trees, and particularly in order to distinguish between pathogenic and non-pathogenic strains of the bacterium, we tested antisera against 40 bacterial cultures with three serological techniques. The serological reactions were very variable. Some of the antisera showed strain specificity whereas others reacted with all strains without any link to the chosen strain or the immunizing protocol. None of the 12 studied antisera was biovar-specific. No serological discrimination was shown between pathogenic and non-pathogenic strains of A tumefaciens. It was concluded that serological diagnosis of the species is not feasible.
\end{abstract}

\section{Agrobacterium tumefaciens / biovar / serology}

Résumé - Quarante souches d'Agrobacterium tumefaciens (dont douze du biovar 1 et vingt-six du biovar 2) ont été étudiées par trois techniques sérologiques dans le but de diagnostiquer l'agent pathogène. Les réactions sérologiques ont été très variables. Certains sérums ont montré une spécificité de souche alors que d'autres réagissaient avec toutes les souches, sans lien apparent entre le choix de la souche et du protocole d'immunisation. Aucun des douze sérums obtenus n'a montré de spécificité vis-à-vis de l'un ou l'autre des deux biovars étudiés. Aucune distinction sérologique n'a été possible entre les souches pathogènes et non pathogènes d'A tumefaciens. Cette étude confirme la grande variabilité antigénique de l'espèce et rejette son diagnostic par la voie sérologique.

Agrobacterium tumefaciens / biovar / sérologie

\section{INTRODUCTION}

La maladie du crown-gall est largement répandue au Maroc où elle a été signalée dans le moyen Atlas, le Gharb, Saïs, Haouz et Tadla.
Elle est particulièrement grave en pépinière dont la plupart sont atteintes à plus de $20 \%$, certaines à plus de $80 \%$. Les principales espèces attaquées sont l'amandier, le pêcher, le prunier, le pommier et le poirier.

\footnotetext{
* Correspondance et tirés à part
} 
Les études sérologiques d'Agrobacterium ont débuté il y a environ un demi-siècle avec pour but l'identification et la classification de la bactérie. Cependant, la diversité du genre Agrobacterium (El Helaly et al, 1969 ; Keane et al, 1970 ; Lopez et al, 1983 ; Zhang et al, 1984 ; Nesme et al, 1987), la complexité de sa structure antigénique (Digat, 1978 ; Süle et El Kady, 1983), et sa large gamme d'hôtes ont compliqué ces études et ont souvent abouti à des résultats contradictoires (Bouzar et al, 1986 ; Alarcon et al, 1987b).

La plupart des études ont révélé une importante variabilité sérologique au sein des biovars d'Agrobacterium définis selon des tests physiologiques. Le genre Agrobacterium a été subdivisé en plusieurs groupes sérologiques dont le nombre dépasse celui des biovars (Berquits et Elrod, 1948 ; Graham, 1971 ; Miller et Vruggink, 1981 ; Moore et Bouzar, 1983 ; Bouzar et al, 1986 ; Nesme et al, 1987). Certains auteurs ont cependant trouvé un grand degré de correspondance entre biovars et sérovars (Keane et al, 1970 ; Lopez, 1978 ; Lopez et al, 1983 ; Alarcon et al, $1987 \mathrm{a}, \mathrm{b}$ ).

Certains auteurs ont tenté de faire la distinction sérologique entre Agrobacterium tumefaciens (souches pathogènes) et Agrobacterium radiobacter (souches non pathogènes) en utilisant différents types d'antigènes et différentes techniques sérologiques. La plupart n'ont pas trouvé de différences entre les deux (Graham, 1971 ; Roberts et Kerr, 1974 ; Lopez, 1978 ; Lopez et al, 1983 ; Alarcon et al, 1987b). Certains ont pu distinguer en double immunodiffusion une souche pathogène et sa variante privée du plasmide $\mathrm{Ti}$ (Hochster et Cole, 1967 ; El Kady et Süle, 1982).

Dans ce travail, nous nous proposons de déterminer les relations sérologiques entre les souches d'Agrobacterium de trois biovars (1, 2 et 1-2) isolés au Maroc et d'étudier la possibilité de diagnostic de l'agent pathogène en utilisant des extraits glycoprotéiques, des bactéries tuées à la chaleur et des bactéries entières mobiles avec trois techniques sérologiques: double immunodiffusion, microagglutination et immunofluorescence indirecte.

\section{MATÉRIEL ET MÉTHODES}

\section{Souches bactériennes et caractérisation biochimique}

Quarante souches d'Agrobacterium tumefaciens sont utilisées dans cette étude (tableau I). Le biovar est déterminé selon le schéma proposé par Benjama et Daoud (1989) (tableau II). Leur pouvoir pathogène est déterminé par inoculation de plantes de tomate, kalanchoe et datura avec des suspensions bactériennes ajustées à environ $10^{8} \mathrm{ufc} / \mathrm{mL}$.

\section{Préparation des antigènes}

Les antigènes «O $\mathrm{O}$ " ou bactéries tuées à $100^{\circ} \mathrm{C}$ pendant 10 minutes sont obtenus selon Raynaud et Digeon (1949) à partir des souches AT 68, 72.18 et 141.5 du biovar 1 et de la souche 103.16 de biovar 2 . Les antigènes «OH» ou bactéries entières mobiles sont obtenus avec des bactéries tuées au formol selon Lopez (1978) à partir des souches 72.18 et $141.5 \mathrm{du}$ biovar 1 et la souche $103.16 \mathrm{du}$ biovar 2. Les antigènes "GP» ou extraits glycoprotéiques sont obtenus selon Digat (1978) à partir des souches 72.18 du biovar 1 et 103.16 du biovar 2 .

\section{Préparation des antisérums}

Douze sérums différents sont préparés à partir des préparations antigéniques décrites plus haut (tableau II). Des lapins pesant 1 à $1,5 \mathrm{~kg}$ sont immunisés par des injections bihebdomadaires en intraveineuse de doses croissantes d'antigène $(0,5 \mathrm{~mL}-1 \mathrm{~mL}$ $-2 \mathrm{~mL}-2 \mathrm{~mL}$ d'une suspension de $10^{10} \mathrm{ufc} / \mathrm{mL}$ ). La saignée des lapins est réalisée 4 à 10 jours après la dernière injection. Les antisérums sont filtrés à travers une membrane Millipore $(0,2 \mu \mathrm{m})$ et stockés à $-70^{\circ} \mathrm{C}$.

\section{Techniques sérologiques}

Pour l'immunodiffusion double (Ouchterlony, 1958), le puits central est rempli d'antisérum à la dilution 1/2. Les puits périphériques sont remplis de suspensions très denses de cultures bactériennes. La formation de lignes de précipitation entre le puits central et les puits périphériques est examinée.

Pour l'immunofluorescence indirecte (Faure et al, 1977), les bactéries sont fixées sur lames de microscope. Le sérum fluorescent antilapin est utilisé au $1 / 100^{e}$. L'observation des lames se fait au grossissement $\times 1000$ à l'immersion avec un microscope à lumière ultraviolette incidente.

Les microagglutinations sont réalisées en plaque de microtitration à cuves à fond rond. Les antisérums sont dilués en tampon phosphate $\left(\mathrm{K}_{2} \mathrm{HPO} 417,41 \mathrm{~g}\right.$; $\mathrm{KH}_{2} \mathrm{PO} 46,545 \mathrm{~g}$; eau distillée $1,500 \mathrm{~L}$; $\mathrm{pH} 7,2$ ) jusqu'à la dilution $1 / 51200^{\circ}$. La suspension bactérienne utilisée est de faible densité $\left(10^{7} \mathrm{ufc} / \mathrm{mL}\right)$. L'observation des plaques se fait au microscope ordinaire au grossissement $\times 40$. Ces tests ont été répétés trois fois. 
Tableau I. Origine et caractéristiques des souches Agrobacterium tumefaciens.

\begin{tabular}{|c|c|c|c|c|c|}
\hline Souches & Origine & Date d'isolement & Plante hôte & Production de tumeur $\mathrm{a}$ & Biovar \\
\hline AT 68 & Grece & 1967 & Prunus amygdalus & + & 1 \\
\hline AT 82143 & France & 1988 & Prunus amygadlus & + & 1 \\
\hline 72.18 & Meknès & 1984 & Prunus domestica & + & 1 \\
\hline 103.36 & Meknès & 1985 & Prunus domestica & + & 1 \\
\hline 141.1 & Azrou & 1987 & Prunus persica & + & 1 \\
\hline 141.5 & Azrou & 1987 & Prunus persica & + & 1 \\
\hline 141.9 & Azrou & 1987 & Prunus persica & + & 1 \\
\hline 208.4 & Meknès & 1989 & Prunus persica & + & 1 \\
\hline 209.3 & Meknès & 1989 & Prunus persica & + & 1 \\
\hline 121.1 & Azrou & 1986 & Prunus amygdalus & + & 2 \\
\hline 121.85 & Azrou & 1986 & Prunus amygdalus & + & 2 \\
\hline 121.90 & Azrou & 1986 & Prunus amygdalus & + & 2 \\
\hline 214.2 & Azrou & 1989 & Prunus amygdalus & + & 2 \\
\hline 214.4 & Azrou & 1989 & Prunus amygdalus & + & 2 \\
\hline 214.16 & Azrou & 1969 & Prunus amygdalus & + & 2 \\
\hline 102.9 & Meknès & 1985 & Prunus domestica & + & 2 \\
\hline 103.16 & Meknès & 1985 & Prunus domestica & + & 2 \\
\hline 103.18 & Meknès & 1985 & Prunus domestica & + & 2 \\
\hline 103.21 & Meknès & 1985 & Prunus domestica & + & 2 \\
\hline 135.5 & Meknès & 1986 & Prunus domestica & + & 2 \\
\hline 141.11 & Azrou & 1987 & Prunus persica & + & 2 \\
\hline 141.12 & Azrou & 1987 & Prunus persica & + & 2 \\
\hline 141.13 & Azrou & 1987 & Prunus persica & + & 2 \\
\hline 142.7 & Azrou & 1987 & Prunus persica & + & 2 \\
\hline 142.8 & Azrou & 1987 & Prunus persica & + & 2 \\
\hline 193.16 & Meknès & 1988 & Prunus persica & + & 2 \\
\hline 193.67 & Meknès & 1988 & Prunus persica & + & 2 \\
\hline 201.12 & Azrou & 1989 & Prunus persica & + & 2 \\
\hline 208.3 & Meknès & 1989 & Prunus persica & + & 2 \\
\hline 121.27 & Azrou & 1986 & Prunus amygdalus & - & 1 \\
\hline 136.4 & Meknès & 1986 & Prunus amygdalus & - & 1 \\
\hline 141.10 & Azrou & 1987 & Prunus persica & - & 1 \\
\hline 142.5 & Azrou & 1987 & Prunus persica & - & 1 \\
\hline 121.20 & Azrou & 1985 & Prunus amygdalus & - & $1-2 b$ \\
\hline 121.35 & Azrou & 1986 & Prunus amygdalus & - & 2 \\
\hline 121.84 & Azrou & 1986 & Prunus amygdalus & - & 2 \\
\hline 121.87 & Azrou & 1986 & Prunus amygdalus & - & 2 \\
\hline 134.2 & Meknès & 1986 & Prunus domestica & - & 2 \\
\hline 134.10 & Meknès & 1986 & Prunus domestica & - & 2 \\
\hline K84 & Australie & 1969 & Sol & - & 2 \\
\hline
\end{tabular}

a Tumeur par inoculation de Datura, Kalanchoe et tomate ; b souche intermédiaire (Benjama et Daoud, 1989).

\section{RÉSULTATS ET DISCUSSION}

Les résultats de cette étude montrent une grande hétérogénéité sérologique des souches marocaines d'Agrobacterium, aussi bien en immunodiffusion double, en microagglutination qu'en immunofluorescence indirecte, ce qui confirme la complexité de la structure antigénique
d'Agrobactérium (Digat, 1978 ; Süle et El Kady, 1983). Ne seront présentés que les résultats obtenus en immunodiffusion.

Les sérums $S 8$ (GP) et $S 21$ (O) réagissent avec toutes les souches d'Agrobacterium par la formation d'une ou plusieurs lignes (tableaux III et (V). Les lignes de précipitation fusionnent entre les souches homologues et hétérologues, ce qui montre leur identité, sans distinction entre 
Tableau II. Titres des onze sérums étudiés et leurs protocoles respectifs d'immunisation.

\begin{tabular}{|c|c|c|c|c|c|c|c|c|c|c|c|c|c|}
\hline \multirow{2}{*}{$\begin{array}{l}\text { Code du } \\
\text { sérum }\end{array}$} & \multirow{2}{*}{$\begin{array}{l}\text { Antigène } \\
\text { de biovar }\end{array}$} & \multirow[t]{2}{*}{ Souche } & \multicolumn{9}{|c|}{ Protocole d'immunisation (jour) } & \multicolumn{2}{|c|}{ Titre } \\
\hline & & & 0 & 2 & 4 & 6 & 10 & 12 & 16 & 18 & 20 & (M agglut) & $(I F)$ \\
\hline-- & - & - & & -- & & & $\cdots$ & & & & & - & \\
\hline S2 & $\mathrm{OH}$ & $72.18 \mathrm{~b} 1$ & 0,5 & 1 & & 2 & 2 & & & & & $1 / 12800$ & $1 / 1000$ \\
\hline S5 & $\mathrm{OH}$ & 72.18 b1 & & & & 2 & 2 & & & & & $1 / 51200$ & $1 / 10000$ \\
\hline S22 & $\mathrm{OH}$ & 103.16 b2 & & & & 1,5 & 1,5 & 1,5 & & & & $1 / 6400$ & $1 / 1000$ \\
\hline S3 & 0 & AT68 b1 & & & & 2 & 2 & & & & & $1 / 12800$ & $1 / 5000$ \\
\hline S4 & 0 & $72.18 \mathrm{~b} 1$ & & & & 2 & 2 & & & & & $1 / 6400$ & $1 / 1000$ \\
\hline S6 & $\mathrm{O}$ & $141.5 \mathrm{~b} 1$ & & & & 2 & 2 & & & & & $1 / 51200$ & $1 / 10000$ \\
\hline S10 & 0 & 141.5 b1 & & & & 2 & 2 & & & & & $1 / 12800$ & $1 / 1000$ \\
\hline S11 & 0 & $72.18 \mathrm{~b} 1$ & & & & 2 & 2 & & & & & $1 / 200$ & $1 / 500$ \\
\hline S21 & $\mathrm{O}$ & $103.16 \mathrm{~b} 2$ & & & & 1,5 & 1,5 & 1,5 & & & & $1 / 12800$ & $1 / 5000$ \\
\hline S8 & GP & 72.18 & & & & 1 & 1 & & & & & $1 / 400$ & $1 / 100$ \\
\hline S20 & GP & $103.16 \mathrm{~b} 2$ & & & & 1,5 & 1,5 & 1,5 & & & & $1 / 25600$ & $1 / 100$ \\
\hline
\end{tabular}

— : injection en intraveineuse d'antigène en $\mathrm{mL} ;-$ : saignée ; b1 : biovar 1 ; b2 : biovar 2 ; IF : immunofluorescence ; M agglut : micro-agglutination.

les biovars ou souches pathogènes et non pathogènes.

Les sérums $\mathrm{S} 20(\mathrm{GP})$ et $\mathrm{S} 22(\mathrm{OH})$ se sont montrés spécifiques seulement de la souche homologue. Un résultat analogue a été obtenu par Miller et Vruggink (1981) et Alarcon et al (1987a, b).

La variabilité est extrême puisque deux sérums répétés avec les mêmes souches et les mêmes protocoles ne fournissent pas les mêmes résultats ( $\mathrm{S} 2$ et $\mathrm{S} 5, \mathrm{~S} 4$ et $\mathrm{S} 11$ dans le tableau III).

Enfin les résultats obtenus en croisant les souches et les sérums montrent l'absence de réciprocité des réactions (tableau IV). Ainsi, la souche 103-16 est reconnue par les autres sérums, mais les anticorps qu'elle a produit lui sont spécifiques.

Aucun cas de spécificité de biovar parmi les douze sérums étudiés. Aucun sérum n'a permis de différencier les souches pathogènes et non pathogènes, ce qui confirme les résultats obtenus par différents auteurs (Graham, 1971 ;
Roberts et Kerr, 1974 ; Miller et Vruggink, 1981 ; Alarcon et al, 1987a, b).

En résumé, les réactions sérologiques vont de la reconnaissance de toutes les souches (S8 et S21), à la reconnaissance d'une seule souche (S20 et S22), en passant par la reconnaissance d'un nombre variable de souches par les sérums S3, S11, S2, S6, S4, S5 et S7, sans différence entre les biovars ou les souches pathogènes ou non pathogènes.

\section{CONCLUSION}

II apparaît donc que la possibilité d'une approche sérologique de l'identification de la bactérie de la galle du collet est à écarter à cause de la grande hétérogénéité sérologique d'Agrobacterium tumefaciens. Le pouvoir pathogène des $A$ tumefaciens sur plantes indicatrices et la technique de détection dans le sol par la PCR (polymerase chain reaction) proposée par Picard et al (1992) restent les moyens fiables de diagnostic de cette espèce. 
Tableau III. Variabilité des réactions d'immunodiffusion double de répétitions de sérums anti- $A$ tumefaciens 72.18 , contre 40 souches des biovars 1 et 2 et 4 souches de Rhizobium.

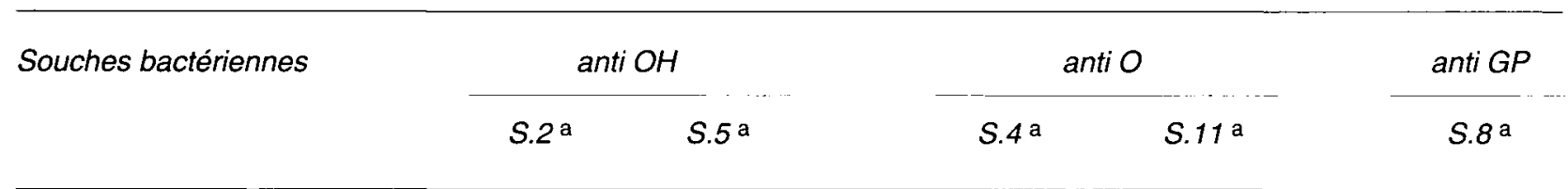

\begin{tabular}{|c|c|c|c|c|}
\hline $\begin{array}{l}\text { A tumefacie } \\
72.18\end{array}$ & (3) & (2) & (1) & (1) \\
\hline AT 68 & $1 \mathrm{~b}$ & 2 & 1 & 0 \\
\hline 141.5 & 2 & 0 & 0 & 1 \\
\hline 141.9 & 2 & 0 & 0 & 1 \\
\hline AT 82143 & 1 & 1 & 1 & 0 \\
\hline 208.4 & 0 & 0 & 1 & 1 \\
\hline 103.86 & 0 & 1 & 0 & 1 \\
\hline 209.3 & 1 & 0 & 0 & 2 \\
\hline 141.1 & 0 & 0 & 0 & 0 \\
\hline
\end{tabular}

Pathogènes bv2

141.13

103.18

214.16

121.90

102.9

141.11

103.16

142.7

142.8

142.8

103.21

193.16

193.67

214.4

141.12

121.85

201.12

208.3

214.2

135.5

$\begin{array}{ll}2 & 0 \\ 2 & 2 \\ 3 & 0 \\ 2 & 0 \\ 2 & 1 \\ 1 & 0 \\ 0 & 2 \\ 1 & 0 \\ 0 & 0 \\ 0 & 0 \\ 0 & 0 \\ 0 & 0 \\ 0 & 2 \\ 0 & 0 \\ 0 & 0 \\ 2 & 0 \\ 0 & 2 \\ 2 & 0 \\ 1 & 0 \\ 0 & 0\end{array}$

(3)

Non pathogènes bv1

141.10

136.4

121.27

142.5

$\begin{array}{ll}3 & 0 \\ 3 & 1 \\ 2 & 0 \\ 0 & 1\end{array}$

Non pathogènes bv2

121.35

121.84

k84

121.87

134.2

134.10

bv $1-2$

121.20

$\begin{array}{ll}2 & 0 \\ 1 & 0 \\ 1 & 1 \\ 0 & 1 \\ 0 & 0 \\ 0 & 0\end{array}$

1
0
1
1
1
0
0
1
1
1
0
0
1
1
1
0
0
1
0
0

2

2

2

3

2

Rhizobium

$\begin{array}{lll}\text { AH } & 0 & 0 \\ \text { AR } & 0 & 0 \\ \text { SG } & 0 & 0 \\ 219.11 & 0 & 0\end{array}$

a Code du sérum ; b nombre de lignes de précipitation ; entre parenthèses réaction homologue. 
Tableau IV. Absence de réciprocité des réactions d'immunodiffusion double de quatre souches $A$ tumefaciens (sérums anti-O).

\begin{tabular}{|c|c|c|c|c|c|c|}
\hline \multirow{2}{*}{$\begin{array}{l}\text { Souches } \\
\text { bactériennes }\end{array}$} & $53^{a}$ & S4 & $S 11$ & S6 & $S 10$ & $S 21$ \\
\hline & anti AT68 & \multicolumn{2}{|c|}{ anti 72.78} & \multicolumn{2}{|c|}{ anti 141.5} & anti 103.16 \\
\hline AT 68 & $3^{b}$ & 1 & 0 & 0 & 0 & 3 \\
\hline 72.18 & 3 & 1 & 1 & 0 & 1 & 2 \\
\hline 141.5 & 3 & 0 & 1 & 3 & 1 & 3 \\
\hline 103.16 & 3 & 0 & 0 & 0 & 0 & 3 \\
\hline
\end{tabular}

a Code du sérum ; b nombre de lignes de précipation.

\section{RÉFÉRENCES}

Alarcon B, Lopez MM, Cambra M, Gorris MT (1987a) Serological study of Agrobacterium biotypes. Proceedings 6th Int Conf on Plant Pathogenic Bacteria, Beltsville, Maryland, 2-7 June

Alarcon B, Lopez MM, Cambra M, Ortiz J (1987b) Comparative study of Agrobacterium biotypes 1, 2 et 3 by electrophoresis and serological methods. $J$ App/ Bacteriol 62, 295-308

Benjama A, Daoud S (1989) Caractérisation de biovars d'isolats marocains d'Agrobacterium issus de tumeurs racinaires des rosacées fruitières. agronomie 9, 887-890

Berquits KR, Elrod RP (1948) The soatic antigens of the genus Agrobacterium. Proc Acad Sci 27, 104111

Bouzar H, Moore LW, Schaad NW (1986) Serological relationship between $50 S$ ribosomal subunits from strains of Agrobacterium and Rhizobium. Phytopathology 76, 1265-1269

Digat B (1978) Antigen specificity in Agrobacterium radiobacter var tumefaciens. Proc 4th Int Conf on Plant Pathogenic Bacteria, Angers, 321-326

El-Helaly AF, Abd-El-Dahab MK, Abd-El-Nil NM (1969) Studies on Egyptian isolates of Agrobacterium tumefaciens ( $\mathrm{Sm}$ et Town) with special reference to their pathological and serological characteristics. Phytopathol Medit 8, 99-106

El Kady S, Süle S (1982) Serological comparison between strains of Agrobacterium radiobacter. Acta Phytopathol Acad Sci Hung 17, 1-6

Faure M, Dupouey P, Morelec MJ (1977) La technique de limmunofluorescence et les réactions immunoenzymatiques. Maloine, Paris, $566 p$

Graham PH (1971) Serological studies with Agrobacterium radiobacter, $A$ tumefaciens and Rhizobium strains. Arch Mikrobiol 78, 70-75

Hochster RM, Cole SE (1967) Serological comparison between strains of Agrobacterium tumefaciens. Can J Microbiol 13, 569-572
Keane PJ, Kerr A, New PB (1970) Crown-gall of stone fruit. II. Identification and nomenclature of Agrobacterium isolates. Aust J Biol Sci 23, 569-572

Lopez MM (1978) Characteristics of French isolates of Agrobacterium. Proc 4th Int Conf on Plant Pathogenic Bacteria, Angers, 233-237

Lopez MM, Micro M, Salcedo CI, Orive RJ, Temprano FJ (1983) Caracteristicas de los aislados espanoles de Agrobacterium radiobacter pv tumefaciens. An Inst Nac Invest Agr, Ser agric 24, 239-249

Miller HJ, Vruggink H (1981) An assessment of biochemical and serological tests for Agrobacterium radiobacter subsp tumefaciens. Phytopathol $Z 102$, 292-230

Moore LW, Bouzar H (1983) Serological relationships of Agrobacterium strains isolated from different ecological niches. 4th Int Cong Plant Pathology, Melbourne (abstract)

Nesme X, Michel MF, Digat B (1987) Population heterogeneity of Agrobacterium tumefaciens in galls of Populus L from a single nursery. App/ Environ Microbiol 53, 655-656

Ouchterlony O (1958) Diffusion in gel methods for immunological analysis. In : Progress in Allergy, Vol 5 (P Kallos, ed), Basel, Karger, 1-78

Picard C, Ponsonnet C, Paget E, Nesme X, Simmonet $P$ (1992) Detection and enumeration of bacteria in soil by direct DNA extraction and polymerase chain reaction. Appl Environ Microbiol 58, 2717-2722

Raynaud M, Digeon M (1949) Sur une nouvelle toxine de bacille typhique extraite des formes rough. $C R$ Acad Sci Paris 229, 564-566

Roberts WP, Kerr A (1974) Crown gall induction: serological reactions, isozyme patterns and sensitivity to mitomycin $\mathrm{C}$ and to bacteriocin of pathogenic strains of Agrobacterium radiobacter. Physiol Plant Pathol 4, 81-91

Süle S, El Kady S (1983) Analysis of plasmid-coded antigens of Agrobacterium radiobacter strain K84. Phytopathol Z 107, 92-95

Zhang J, Na S, Yu M, Xiang W (1984) Identification of several strains isolated from the vicinity of Beijing China. Acta Microbiol Sin 24, 369-375 